Epidemiology and Infection

cambridge.org/hyg

\section{Original Paper}

Cite this article: Mellou K, SarantiPapasaranti E, Mandilara G, Georgakopoulou T (2019). Laboratory capacity of Greek hospitals for diagnosis of salmonellosis and surveillance systems' performance in the years of economic crisis, 2010-2016. Epidemiology and Infection 147, e17, 1-7. https://doi.org/ $10.1017 /$ S0950268818002686

Received: 13 April 2018

Revised: 23 August 2018

Accepted: 4 September 2018

Key words:

Laboratory capacity; notification rate; salmonellosis; surveillance system; underreporting

Author for correspondence:

K. Mellou, E-mail: kmellou@gmail.com

\title{
Laboratory capacity of Greek hospitals for diagnosis of salmonellosis and surveillance systems' performance in the years of economic crisis, 2010-2016
}

\section{K. Mellou ${ }^{1}$, E. Saranti-Papasaranti ${ }^{1}$, G. Mandilara ${ }^{2}$ and T. Georgakopoulou ${ }^{1}$}

${ }^{1}$ Hellenic Centre for Disease Control and Prevention, Athens, Greece and ${ }^{2}$ National Reference Centre for Salmonella, National School of Public Health, Central Public Health Laboratory, Hellenic Centre for Disease Control and Prevention, Vari, Attica, Greece (c) The Author(s) 2018. This is an Open Access article, distributed under the terms of the Creative Commons Attribution licence (http:// creativecommons.org/licenses/by/4.0/), which permits unrestricted re-use, distribution, and reproduction in any medium, provided the original work is properly cited.

\begin{abstract}
Austerity might have affected the capacity of public hospitals in Greece to diagnose salmonellosis (laboratory capacity) over the period 2010-2016, as well as the performance of the existing surveillance systems. The scope of this paper is to present data on laboratory capacity over these years, as well as the results of a two-source capture-recapture study (data from Mandatory Notification System and National Reference Laboratory System for Salmonella). The main findings were that: (a) laboratory capacity was high and steady besides the financial crisis, (b) the estimated number of laboratory-confirmed cases ( $n=6017$, 95\% CI 5892-6142) resulted in an incidence rate $(7.9$ cases $/ 100000$ population) almost twice than that reported by the two systems Mandatory Notification System (MNS); 4.1 and National Reference Laboratory System (NRLS); 4.5 cases/100 000 population, (c) underreporting was high for both systems (MNS; $47.5 \%$ and NRLS; $42.8 \%$ ) and (d) differences by geographical region, size and type of hospital were identified. We suggest that (a) specific interventions are needed to increase completeness of the systems by type of hospital and geographical region, (b) record linkage can help in estimating the disease burden in a more valid way than each system separately and (c) a common electronic database in order to feed one system to the other could significantly increase completeness of both systems.
\end{abstract}

\section{Introduction}

Salmonellosis is a foodborne disease caused by Salmonella spp. that results in a high global morbidity and mortality. The World Health Organization estimated that non-typhoidal Salmonella spp. caused 78 million cases of foodborne illness, 28693 deaths and 2183146 disability-adjusted life years globally in 2010 [1]. According to the European Food Safety Authority and the European Centre for Disease Prevention and Control, the EU/EEA annual salmonellosis notification rate for confirmed cases during the period 2010-2016 ranged from 20.3 to 21.9 per 100000 population [2-4].

In Greece, there are two parallel surveillance systems for salmonellosis, both paper-based; Mandatory Notification System (MNS) for clinical cases and surveillance through the National Reference Laboratory System for Salmonella (NRLS), which is voluntary, but universal. Based on the MNS data, the mean annual notification rate of salmonellosis for the period 2010-2016 was 4.1 cases per 100000 population (s.D. 1.37), having an increasing trend after 2014 [5].

There are limitations associated with the use of data from these surveillance systems if the degree of underreporting is not addressed [6]. Quantification of underreporting is needed in order to estimate the actual burden of the disease in the country, detect outbreaks early and evaluate policies for improving food safety [7, 8]. Since 2010 several efforts have been made to improve disease notification by medical doctors (in terms of routine feedback to hospitals regarding the management of cases/outbreaks of salmonellosis, publications emphasising the importance of notification, etc.) $[9,10]$.

On the other hand, during the same period, the country was in the middle of a financial crisis that began in November 2009 but deepened after the implementation of austerity measures in early 2010.

According to the Organization for Economic Co-operation and Development (OECD), health care spending in Greece has been consistently decreasing over the last years (total spending per capita in 2010 was USD 2696 and in 2016 was USD 2223), following the financial crisis [11]. The health care system, which is a highly centralised mixed model, incorporating both tax-based financing and social health insurance has experienced the consequences [12]. Strong annual growth increases were reversed after 2009 and there have been significant cuts in health spending (5.4\% for the period 2003-2009 vs. $-5.0 \%$ for the period 2009-2016) [11, 13]. 
Still, the national health system provides universe coverage to the population. Greek residents including uninsured people, legal and illegal migrants can visit the emergency rooms of the public hospitals, free of charge.

In Greece, the spending to GDP ratio has fluctuated, approaching close to $10 \%$ in 2010 , before returning at around $8 \%$ of GDP in the following years [11]. In 2016, the USA spent more than eight percentage points above the OECD average $(9.0 \%$ of Gross Domestic Product (GDP)) on health [11, 14]. A group of ten highincome OECD countries, including Switzerland, Germany, Sweden, France, Japan and Canada followed with about 11\% of GDP going to health services $[11,14,15]$. Studies have demonstrated that service quality of public hospitals in Greece deteriorated during this period and that the decrease of the health care spending has resulted in increased workload for medical staff [16-20]. Additionally, the financial crisis might have also affected the capacity of the Greek hospitals to diagnose salmonellosis cases (perform cultures).

The objective of this paper is to: (a) investigate whether there have been significant changes in the laboratory capacity of Greek hospitals to diagnose salmonellosis over the period 2010-2016, (b) estimate the actual number of laboratory-confirmed salmonellosis cases at Greek hospitals during the same period, (c) assess underreporting of salmonellosis to both existing surveillance systems, (d) investigate whether there have been significant changes of underreporting rate over the years and (e) identify factors related to underreporting rate in each one of the surveillance systems in order to propose appropriate strategies for the improvement of the systems' completeness.

\section{Methods}

\section{Laboratory capacity}

Since 2010 the Department of Epidemiological Surveillance and Intervention of Hellenic Centre for Disease Control and Prevention (HCDCP) collects data on the capacity of the microbiological laboratories of the public hospitals of the country to diagnose foodborne diseases. Each year, in January an official letter is sent to the administration of hospitals requesting for information on the current capacity of the hospital's laboratory to perform testing for a series of pathogens, including Salmonella spp. Hospitals are asked to complete a structured form and sent it back to HCDCP within 3 weeks. The official letter accompanying the request for data explains that one of the benefits from this recording is to have evidence on important changes of laboratory capacity compared with previous years and be able to assess to some degree the under-ascertainment rate of foodborne diseases in the country, which is an important element, for assessing the diseases' burden. Additionally, changes of 'laboratory capacity' of the hospitals are directly linked to changes of the recorded notification rate for reasons other than an actual change of the morbidity.

After the 3 weeks time, the information is usually requested again from the hospitals that have not yet replied and an extension is given for the collection of data. Data are recorded in a specially designed database, are analysed and the report is sent to relevant stakeholders (directors of hospitals, Ministry of Health, etc.). Usually, the aforementioned work is finalised by the end of March each year.

The overall laboratory capacity of public hospitals to diagnose salmonellosis is calculated by dividing the number of hospitals that routinely perform cultures for Salmonella spp. by the total number of hospitals for which the information is available.

\section{Capture-recapture study}

The two-source capture-recapture method was employed on the basis of salmonellosis notifications from NRLS and MNS [21, 22]. Clinical doctors notify new salmonellosis cases to local public health authorities and HCDCP through the MNS. In specific, after the laboratory confirmation of the case (isolation of Salmonella spp. from stool, urine, body site (e.g. infected wound) or any normally sterile body fluids and tissues (e.g. blood, CSF, bone, synovial fluid, etc.)) doctors complete the respective notification form of MNS that contains the name and demographic characteristics of the cases (sex, date of birth, place of residence), clinical symptoms, date of notification and laboratory data. Reported cases are classified in accordance with the European case definition and only cases with at least one of the following four; diarrhoea, fever, abdominal pain, vomiting are recorded [5].

In parallel with the notification of cases from clinical doctors, microbiologists are requested to send Salmonella spp. isolates to the national reference center located in Attica region for further typing (NRLS system). Each isolate is accompanied by a short form that includes the name and demographic characteristics (name, age, sex, date of birth, region) of the patient and the date of specimen collection. Isolates are serotyped and results are sent to the microbiological laboratories of the hospitals. Further molecular typing is also performed, in the case of clusters/outbreaks.

The purposes of the two systems are common but not identical. Both systems aim at monitoring the temporal distribution of salmonellosis and early detection of outbreaks, however, an additional aim of NRLS is to follow the trends of specific serotypes, identify emerging serotypes and proceed to molecular techniques for the identification of open outbreaks.

Information from the two sources was merged to estimate the real number of cases and the completeness of each source. All salmonellosis cases (non-typhoidal) reported during the period 2010-2016 at any of the two surveillance systems were included in the study. Data were checked for duplicates and in case there were two or more isolates from the same patient only the first one was included in the analysis. Isolates from asymptomatic cases were excluded from the database of NRLS.

A joint database was generated in order to include all reported cases of both systems. The detection of the same individuals in the two sources was determined by name, sex, age, date of notification and reporting hospital.

\section{Statistical analysis}

The actual number of laboratory-confirmed cases $(N)$ and the respective 95\% confidence intervals (95\% CI) were estimated with the use of (a) Chapman's formula and (b) Chao's lower bound estimator for a two-source capture-recapture data $[23,24]$.

Chapman's formula is traditionally used in capture-recapture studies with two sources [21]. However, the results of the analysis could be biased if the two data sources are positively dependent, thus generating underestimation of cases. Chao's lower bound estimator has been shown to be less affected by source dependence and provides more reliable estimates unless both sources are independent. Odds ratio (OR) would be close to unity when the two sources are independent [22]. In order to compute the $\mathrm{OR}$, we estimated the number of individuals that did not appear in either source $\left(f_{00}\right)$ (Table 1) [23]. The estimate is given by 
Table 1. The two-source situation

\begin{tabular}{lllll}
\hline Source 1 & & 1 & 0 & \\
\hline Source 2 & 1 & $f_{11}$ & $f_{01}$ & $L_{2}$ \\
\hline & 0 & $f_{10}$ & $f_{00}$ & \\
\hline & & $L_{1}$ & & \\
\hline
\end{tabular}

the formula: $\widehat{f_{000}}=\frac{f_{10} f_{01}}{f_{11}+1}$. Underreporting of salmonellosis to each system was estimated by dividing the reported number of cases by the estimated number of laboratory-confirmed cases, as determined by capture-recapture analysis using both estimators. Underreporting rate of salmonellosis was also estimated by year and month of notification, type of hospital (public/private), hospital beds $(<250 / \geqslant 251)$ and geographical region of the country (Attica/rest of Greece). Also, underreporting rates and these factors possibly affecting notification were compared between the two notification systems.

The $t$ test and Mann-Whitney $U$ test were performed to compare the mean rates between two independent groups. Furthermore, a test of linear trend was performed, after computing underreporting rates for each year of follow up, by setting up regression analysis for testing the statistical significance of the seasonality [25].

The statistical analysis was performed using Microsoft Excel and STATA statistical software and $P$-values less than 0.05 were considered statistically significant.

\section{Ethics}

The HCDCP is the competent authority for surveillance of communicable diseases according to Greek legislation and has been officially authorised to receive, treat and temporarily store personal data of infectious diseases cases by the Greek Authority for Personal Data Protection. Personal data were used only for the purposes of the matching procedure. All the necessary measures to protect the confidentiality of personal data were taken during the whole process. Access to the data was restricted to the personnel involved in data analysis and personal data were removed from the datasets after matching.

\section{Results}

\section{Laboratory capacity}

Based on the hospital records, as described in Table 2, the overall laboratory capacity of the Greek hospitals to perform cultures and diagnose salmonellosis was high (more than $80 \%$ ), for the period 2010-2016.

\section{Estimation of the actual number of cases and of underreporting rates}

During the 7-year study period, 3161 salmonellosis cases were notified to MNS, 3444 cases to NRLS and 1809 cases were notified to both systems (Table 3 ), resulting to a mean annual notification rate of 4.1 and 4.5 cases per 100000 population, respectively. The estimated actual number of laboratory-confirmed salmonellosis cases at Greek hospitals, for the period 2010-2016, was 6017 (95\% CI 5892-6142), resulting to a mean annual incidence rate
Table 2. Capacity of microbiological laboratories of public hospitals to perform cultures for Salmonella spp., Greece, 2010-2016

\begin{tabular}{|c|c|c|c|c|c|c|c|}
\hline & 2010 & 2011 & 2012 & 2013 & 2014 & 2015 & 2016 \\
\hline $\begin{array}{l}\text { Capacity to } \\
\text { perform } \\
\text { cultures for } \\
\text { Salmonella } \\
\text { spp. }^{\text {a }}\end{array}$ & $\begin{array}{r}100 / 115 \\
(87 \%)\end{array}$ & $\begin{array}{r}102 / 116 \\
(88 \%)\end{array}$ & $\begin{array}{c}81 / 89 \\
(91 \%)^{b}\end{array}$ & $\begin{array}{l}76 / 87 \\
(87 \%)\end{array}$ & $\begin{array}{l}67 / 83 \\
(81 \%)\end{array}$ & $\begin{array}{l}75 / 87 \\
(86 \%)\end{array}$ & $\begin{array}{l}82 / 89 \\
(92 \%)\end{array}$ \\
\hline
\end{tabular}

${ }^{a}$ Number of hospitals that routinely perform cultures for Salmonella spp. divided by the total number of hospitals for which the information was available.

${ }^{b}$ Drop of the number of hospitals after 2011 had mostly to do with the fact that some hospitals were closed or merged with others of the same area.

of 7.9 cases per 100000 population. The salmonellosis notification rate and estimated incidence rate from the MNS and the NRLS for the period 2010-2016 is depicted in Figure 1.

As far as the estimated underreporting of the MNS and NRLS system is concerned, it was $47.5 \%$ and $42.8 \%$, respectively, for salmonellosis cases. As shown in Table 3, estimates of cases were not very different using both formulae, since the two sources were independent (OR 1, 95\% CI 0.9-1.1) and thus for convenience reasons only estimators as determined by Chapman's formula are included in the text.

\section{Underreporting rates over the years and seasonal trend}

Regarding the underreporting rate of salmonellosis to MNS there was a statistically significant decreasing trend $(P=0.002)$ over the period 2010-2016, while in NRLS system no significant trend in underreporting was observed $(P=0.212)$ (Fig. 2). For both notification systems, even though there was no statistically significant seasonal trend observed (MNS: $P=0.087$, NRLS: $P=0.169$ ), the mean annual underreporting rates increased during winter months reaching a peak in December and gradually decreased in summer (Table 3).

\section{Factors affecting MNS and NRLS notification rates}

In MNS, private hospitals and hospitals located in Attica had significantly higher salmonellosis underreporting rates than public and district hospitals $(P=0.048$ and $P=0.025$, respectively), while in NRLS system, public, district and small hospitals had significantly higher underreporting rates than private and large hospitals in Attica $(P=0.002, P=0.002$ and $P=0.029$, respectively) (Table 3 ).

\section{Comparison of the two systems}

The two sources were independent since the odds ratio was close to unity; therefore, comparison of the two notification systems was possible. Salmonellosis underreporting of MNS was significantly higher compared with underreporting of NRLS throughout the study period. Also, MNS had statistically significant higher underreporting rates from private hospitals compared with the NRLS system $(P=0.001)$ (Table 4$)$.

\section{Discussion}

Knowledge of the epidemiology of foodborne diseases is vital for planning, implementing and evaluating public health policies and practices $[7,26]$. In order to be able to estimate the actual burden 


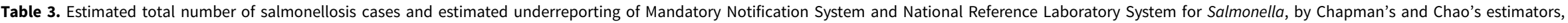
Greece, 2010-2016

\begin{tabular}{|c|c|c|c|c|c|c|c|c|c|}
\hline & \multirow[b]{2}{*}{$\begin{array}{l}\text { No. records } \\
\text { in MNS }\end{array}$} & \multirow[b]{2}{*}{$\begin{array}{l}\text { No. records } \\
\text { in NRLS }\end{array}$} & \multirow[b]{2}{*}{$\begin{array}{l}\text { Matched } \\
\text { records }\end{array}$} & \multicolumn{3}{|c|}{ Total no. of estimated cases (Chapman's formula) } & \multicolumn{3}{|c|}{ Total no. of estimated cases (Chao's formula) } \\
\hline & & & & $\begin{array}{l}\text { Total cases } \\
(N)(95 \% \mathrm{Cl})\end{array}$ & $\begin{array}{c}\text { MNS } \\
\text { underreporting (\%) }\end{array}$ & $\begin{array}{c}\text { NRLS } \\
\text { underreporting (\%) }\end{array}$ & $\begin{array}{l}\text { Total cases } \\
(\mathrm{N})(95 \% \mathrm{Cl})\end{array}$ & $\begin{array}{c}\text { MNS } \\
\text { underreporting (\%) }\end{array}$ & $\begin{array}{c}\text { NRLS } \\
\text { underreporting (\%) }\end{array}$ \\
\hline All cases & 3161 & 3444 & 1809 & 6017 (5892-6142) & 47.5 & 42.8 & $6086(5951-6221)$ & 47.6 & 42.9 \\
\hline \multicolumn{10}{|l|}{ By year } \\
\hline 2010 & 301 & 386 & 155 & $748(685-811)$ & 59.8 & 48.4 & $761(695-827)$ & 60.5 & 49.3 \\
\hline 2011 & 475 & 570 & 287 & $943(895-991)$ & 49.6 & 39.5 & $951(902-1001)$ & 50.1 & 40.1 \\
\hline 2012 & 404 & 433 & 211 & $828(773-883)$ & 51.2 & 47.7 & $830(775-886)$ & 51.3 & 47.8 \\
\hline 2013 & 419 & 397 & 202 & $822(766-879)$ & 49.1 & 51.7 & $824(767-881)$ & 49.2 & 51.8 \\
\hline 2014 & 348 & 384 & 206 & $648(610-686)$ & 46.3 & 40.8 & $650(611-689)$ & 46.5 & 40.9 \\
\hline 2015 & 467 & 528 & 294 & $838(800-877)$ & 44.3 & 37.0 & $842(802-881)$ & 44.5 & 37.3 \\
\hline 2016 & 747 & 746 & 454 & $1227(1183-1271)$ & 39.1 & 39.2 & $1227(1183-1272)$ & 39.1 & 39.2 \\
\hline \multicolumn{10}{|l|}{ By type of hospital } \\
\hline Public & 2735 & 2620 & 1440 & 4975 (4857-5094) & 45.0 & 47.3 & $4978(4860-5097)$ & 45.1 & 47.4 \\
\hline Private & 419 & 796 & 369 & 904 (880-927) & 53.6 & 11.9 & $1000(960-1040)$ & 58.1 & 20.4 \\
\hline \multicolumn{10}{|l|}{ By hospital beds } \\
\hline$<250$ & 1310 & 1147 & 673 & $2232(2157-2307)$ & 41.3 & 48.6 & 2243 (2166-2319) & 41.6 & 48.9 \\
\hline$\geqslant 251$ & 1785 & 2057 & 1104 & $3325(3243-3408)$ & 46.3 & 38.1 & $3343(3259-3426)$ & 46.6 & 38.5 \\
\hline \multicolumn{10}{|l|}{ By region } \\
\hline Attica & 1263 & 2012 & 992 & $2561(2509-2614)$ & 50.7 & 21.4 & 2703 (2637-2769) & 53.3 & 25.6 \\
\hline Rest of Greece & 1891 & 1404 & 817 & $3249(3140-3357)$ & 41.8 & 56.8 & $3322(3207-3437)$ & 43.1 & 57.7 \\
\hline \multicolumn{10}{|c|}{ By month of notification } \\
\hline January & 120 & 123 & 57 & $258(223-292)$ & 53.4 & 52.3 & $259(223-295)$ & 53.7 & 52.5 \\
\hline February & 101 & 103 & 55 & $188(166-211)$ & 46.4 & 45.3 & $189(166-212)$ & 46.6 & 45.5 \\
\hline March & 107 & 113 & 65 & $186(167-204)$ & 42.3 & 39.1 & $186(168-205)$ & 42.5 & 39.3 \\
\hline April & 153 & 182 & 100 & 278 (257-299) & 45.0 & 34.5 & $281(258-303)$ & 45.5 & 35.1 \\
\hline May & 210 & 220 & 126 & 366 (340-392) & 42.7 & 39.9 & 367 (340-393) & 42.8 & 40.0 \\
\hline June & 282 & 288 & 173 & 469 (442-496) & 39.9 & 38.6 & $470(442-497)$ & 39.9 & 38.7 \\
\hline July & 405 & 448 & 246 & 737 (698-776) & 45.0 & 39.2 & 739 (700-779) & 45.2 & 39.4 \\
\hline August & 562 & 661 & 359 & 1034 (991-1078) & 45.7 & 36.1 & $1042(997-1086)$ & 46.0 & 36.5 \\
\hline September & 465 & 523 & 278 & 874 (830-919) & 46.8 & 40.2 & 878 (883-923) & 47.0 & 40.4 \\
\hline October & 344 & 365 & 182 & 689 (641-737) & 50.1 & 47.0 & $690(642-739)$ & 50.2 & 47.1 \\
\hline November & 261 & 271 & 122 & 578 (523-633) & 54.9 & 53.1 & $580(524-636)$ & 55.0 & 53.3 \\
\hline December & 151 & 134 & 46 & 436 (353-518) & 65.3 & 69.2 & $441(355-528)$ & 65.8 & 69.6 \\
\hline
\end{tabular}




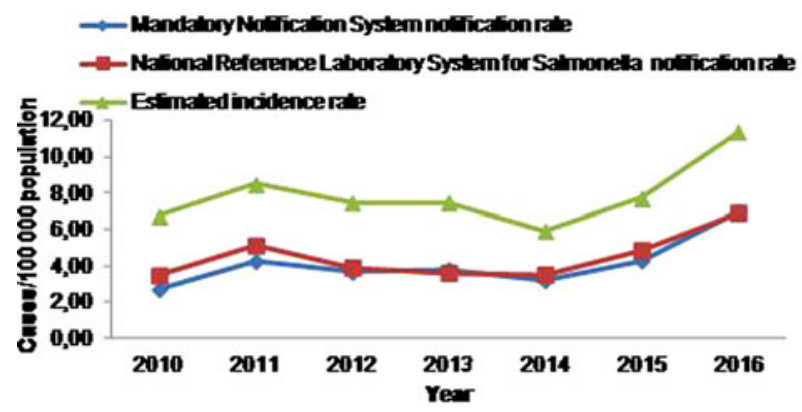

Fig. 1. Time trend of salmonellosis notification rate at the Mandatory Notification System and the National Reference Laboratory System for Salmonella and estimated incidence rate, Greece, 2010-2016.

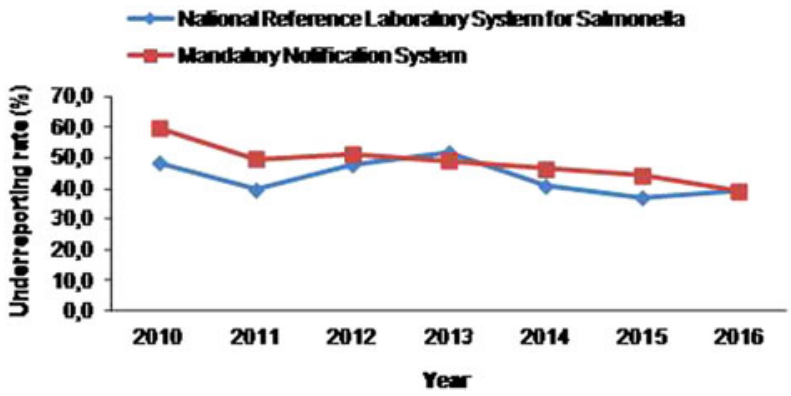

Fig. 2. Trend of underreporting rate of salmonellosis at the Mandatory Notification System and the National Reference Laboratory System for Salmonella by year, 2010-2016.

of a disease in the country, data on the laboratory capacity of medical services and underreporting rates to surveillance systems are needed, amongst others $[6,27]$. In Greece, there is a lack of published literature regarding the performance of surveillance systems of foodborne diseases, thus the estimation of their actual burden is difficult [9].

Based on the hospital records, besides the financial crisis, laboratory capacity of the Greek hospitals to perform cultures and diagnose salmonellosis was high and steady throughout the study period.

Capture-recapture analysis is a useful and not costly way to estimate the actual number of laboratory-confirmed cases at Greek hospitals, to evaluate salmonellosis underreporting rate with accuracy, to interpret collected data appropriately, to identify weaknesses of surveillance systems and to indicate factors associated with underreporting rate in order to apply correction measures for the improvement of the system's completeness [28, 29].

A well-known limitation of capture-recapture methods is their sensitivity to their underlying four assumptions. Authors believe that assumptions were not seriously violated in this study: (1) the study population was closed, (2) all cases had the same probability of being captured to each of the systems (catchability), (3) cases of the surveillance systems were identified and matched and (4) there was independence between the two sources [23, 24, 30, 31].

According to the results of this capture-recapture study, the actual number of laboratory-confirmed cases at Greek hospitals was substantially higher than that reported from MNS and NRLS system, resulting in an estimated incidence rate almost twice than that reported. Consequently, notification rates substantially
Table 4. Underreporting rates by year and month of notification, type and size of hospital and geographical region; Mandatory Notification System and National Reference Laboratory System for Salmonella, Greece, 2010-2016

\begin{tabular}{|c|c|c|c|}
\hline & \multicolumn{3}{|c|}{ Salmonella spp. } \\
\hline & & $\begin{array}{l}\text { Underreporting \% } \\
\text { (Mean rates) }\end{array}$ & $P$-value \\
\hline \multirow[t]{2}{*}{ Year of notification } & MNS & 48.5 & 0.147 \\
\hline & NRLS & 43.5 & \\
\hline \multirow{2}{*}{$\begin{array}{l}\text { Month of } \\
\text { notification }\end{array}$} & MNS & 48.1 & 0.313 \\
\hline & NRLS & 44.5 & \\
\hline \multirow{2}{*}{$\begin{array}{l}\text { Type of hospital } \\
\text { (public/private) }\end{array}$} & MNS & 51.0 & 0.001 \\
\hline & NRLS & 30.2 & \\
\hline \multirow{2}{*}{$\begin{array}{l}\text { Hospital beds } \\
(<250 / \geqslant 251)\end{array}$} & MNS & 44.4 & 0.894 \\
\hline & NRLS & 44.0 & \\
\hline \multirow{2}{*}{$\begin{array}{l}\text { Region (Attica/Rest } \\
\text { of Greece) }\end{array}$} & MNS & 46.7 & 0.243 \\
\hline & NRLS & 40.0 & \\
\hline
\end{tabular}

underestimate the actual number of laboratory-confirmed salmonellosis cases at Greek hospitals.

Furthermore, the underreporting rate of salmonellosis in Greece decreased during the period 2010-2016 for both surveillance systems in place. Sensitisation initiatives of HCDCP since 2010 seem to have contributed to this decrease. Estimated underreporting of salmonellosis in this study is similar to previous estimations in the country $[9,32,33]$ and to estimations of reporting rates in Spain [34]. Also, austerity measures did not seem to have deteriorated the completeness of surveillance systems for salmonellosis even though they might have decreased the degree of potential improvement due to the initiatives of the last years.

On the other hand, underreporting of salmonellosis remains higher than that reported from other European countries (e.g. Sweden, [35], Germany [36], Italy [36], Ireland [37]) depicting that reporting has not been optimal and new strategies are needed.

In Greece, a significant seasonal trend is observed for notified salmonellosis cases, with the mean annual notification rate increasing during summer, reaching a peak in August and gradually decreasing in autumn [5], which is a seasonality pattern consistent with findings from other European countries (Malta, Poland, Portugal, Romania, Slovakia and Spain) [2]. Underreporting rate seems to follow a pattern reciprocal to the seasonality pattern of notification rate. Higher undernotification in winter months, with a peak in December, can be explained by a reduced alertness of the clinicians to notify cases when the disease is less frequent and consequently perceived as not important from a public health point of view [32].

Analysis of underreporting at MNS showed that hospitals located at the capital notify less frequently. This finding may be attributed to the increased workload since almost $50 \%$ of the population of the country resides in Attica and also to the constant changing of personnel working at the hospitals in the capital. These findings are in accordance with other studies [6, 37-40], showing that excess work, lack of time and proper training of personnel on disease reporting are associated with increased underreporting rates. On the contrary, analysis of underreporting of NRLS showed that large hospitals located in Attica send isolates to the reference centre more, probably due to the lower transportation cost, compared with hospitals located in other regions. Also, 
larger hospitals probably have a better mechanism in place in order to cover the cost of transportation to the reference centre than smaller ones.

The fact that estimated underreporting for salmonellosis, in MNS, was statistically significantly higher for private hospitals and that in NRLS system it was higher for public hospitals, might reflect the different manners that public and private hospitals use the different systems; it has been observed that private hospitals make use of the system for providing more information to their clients, while clinicians of public hospitals do not consider the serotype as a clinically relevant information and avoid sending the isolates to the reference lab, especially for sporadic cases.

A striking finding is that the underreporting rate of MNS, which is a compulsory system, is higher than the underreporting rate of NRLS system, which is a voluntary system. This result shows that clinicians working at the hospitals notify less new salmonellosis cases through MNS compared with microbiologists. Inadequate training of clinical doctors on the importance of surveillance systems and on the basic public health concepts and principles may be a possible explanation of this difference, however further investigation of the reasons that clinical doctors do not report as systematically as microbiologists are needed.

Ideally, all cases should be notified and the respective isolates to be sent to the reference laboratory in order to have the full completeness of the two systems. Apparently, based on the results of our study this is not the case and we need to increase the completeness of both systems. In order to have more valid data regarding the actual number of salmonellosis cases diagnosed at Greek hospitals, we can conclude that data from the two systems should be combined. The existence of two or more parallel systems for the surveillance of salmonellosis is common in Europe and allows each system to complement the other while it enables crossover conclusions drawn from the analysis of the information collected by each system separately [35]. Record-linkage has been proved to be an important tool for assessing the quality and completeness of registers and in several European countries, clinical and laboratory notifications are combined in order to meet these objectives [35, 41].

Following the example of other European countries, we recommend that the implementation of a common electronic database for both systems that will allow for each system to systematically feed the other is needed in Greece. In this way, when an isolate is sent to the reference laboratory automatically a mandatory notification form will be required from the clinical doctor with a reminder that notification of the disease is mandatory. Additionally, when a mandatory notification form is sent a reminder for sending the isolate to the reference laboratory with practical guidelines may help in increasing completeness of NRLS, too.

In conclusion, the underreporting rate of confirmed salmonellosis cases decreased during the follow-up period (2010-2016) besides the financial crisis, however underreporting of both systems is still high. Differences in the underreporting rate by geographical region, size and type of hospital can guide interventions for the improvement of notification. Efforts to make clinical doctors more aware of reporting to MNS should be continuous, especially for doctors working at private hospitals located in Attica, while for NRLS, efforts should focus at smaller public hospitals in other regions and transportation cost of isolates should be addressed. Record linkage and use of a common electronic database in order one system to feed the other could significantly increase the completeness of both systems.
Investing in better surveillance systems and public health interventions is a cost-saving approach in austerity situations since it is the only way to early detect public health threats and implement preventive measures and thus reduce the cost for health care services (hospitalisation, medication, etc) [42].

\section{Author contributions}

KM conceived of the study and its design, led the study organisation, coordination, data collection and interpretation and contributed to the data analysis, as well as the manuscript's first draft and revisions. ESP participated in the design of the study, collection and entry of the data, performed the statistical analysis and drafted the manuscript. GM participated in the study design, contributed to the data analysis, interpretation and the review of the manuscript. TG reviewed the background, the methodology, the interpretation and the manuscript. All authors read and approved the final manuscript.

Conflict of interest. None.

\section{References}

1. World Health Organization (WHO) (2015) WHO estimates of the Global Burden of Foodborne Diseases: Foodborne Disease Burden Epidemiology Reference Group 2007-2015. World Health Organization. Available at http://apps.who.int/iris/bitstream/10665/199350/1/9789241565165_eng.pdf? ua $=1$ (Accessed 5 February 2018).

2. European Food Safety Authority (EFSA) and European Centre for Disease Prevention and Control (ECDC) (2017) The European Union summary report on trends and sources of zoonoses, zoonotic agents and food-borne outbreaks in 2016. EFSA Journal 15, 5077.

3. European Food Safety Authority (EFSA) and European Centre for Disease Prevention and Control (ECDC) (2012) The European Union summary report on trends and sources of zoonoses, zoonotic agents and food-borne outbreaks in 2010. EFSA Journal 10, 2597.

4. European Centre for Disease Prevention and Control (ECDC) (2016) Surveillance atlas of infectious diseases: salmonellosis. Current Time Period. Available at http://atlas.ecdc.europa.eu/public/index.aspx (Accessed 22 January 2017).

5. Hellenic Centre for Disease Control and Prevention (HCDCP) Epidemiological data for salmonelosis (non typhoid/paratyphoid) in Greece 2004-2016. Mandatory Notification System and National Reference Centre for Salmonella and Shigella. Department of Epidemiological Surveillance and Intervention. Available at http://www.keelpno.gr/Portals/ 0/Files/English\%20files/Reports\%20(annual-periodical)/2016/Epidemiological\% 20data\%20for\%20Salmonellosis_2004-2016.pdf (Accessed 5 February 2018).

6. Gibbons CL et al. (2014) Measuring underreporting and underascertainment in infectious disease datasets: a comparison of methods. BMC Public Health 14, 147. Published online: 11 February 2014.

7. World Health Organization (WHO) (2006) Communicable disease surveillance and response systems: guide to monitoring and evaluating. World Health Organization. Available at http://www.who.int/csr/resources/ publications/surveillance/WHO_CDS_EPR_LYO_2006_2.pdf (Accessed 9 January 2018).

8. Quade P and Nsoesie EO (2017) A platform for crowdsourced foodborne illness surveillance: description of users and reports. JMIR Public Health and Surveillance 3, e42. Published online: 5 July 2017.

9. Mellou K et al. (2013) Evaluation of underreporting of salmonellosis and shigellosis hospitalised cases in Greece, 2011: results of a capture-recapture study and a hospital registry review. BMC Public Health 13, 875. Published online: 23 September 2013.

10. European Centre for Disease Prevention and Control (ECDC) Salmonellosis: just the tip of the iceberg. Available at https://ecdc.europa. eu/en/publications-data/salmonellosis-just-tip-iceberg (Accessed 25 January 2018). 
11. Organization for Economic Co-operation and Development (OECD) (2017) Health at a Glance 2017: OECD Indicators. Paris: OECD Publishing (Accessed 22 February 2018).

12. Economou C et al. (2017) Greece: health system review. Health Systems in Transition 19, 1-166. Published online: 5 July 2018.

13. Stuckler D et al. (2017) Austerity and health: the impact in the UK and Europe. European Journal of Public Health 27, 18-21. Published online: 10 October 2017

14. Papanicolas I, Woskie LR and Jha AK (2018) Health care spending in the United States and other high-income countries. JAMA 319, 1024-1039. Published online: 13 March 2018. doi: 10.1001/jama.2018.1150.

15. Thomson S et al. (2014) Economic Crisis, Health Systems and Health in Europe: Impact and Implications for Policy. WHO Regional Office for Europe. Available at http://www.euro.who.int/_data/assets/pdf_file/0008/ 257579/Economic-crisis-health-systems-Europe-impact-implications-policy. pdf (Accessed 29 June 2018).

16. Keramidou I and Triantafyllopoulos L (2018) The impact of the financial crisis and austerity policies on the service quality of public hospitals in Greece. Health Policy 122, 352-358. Published online: 24 December 2017.

17. Correia T, Dussault G and Pontes C (2015) The impact of the financial crisis on human resources for health policies in three southern-Europe countries. Health Policy 119, 1600-1605.

18. Simou E and Koutsogeorgou E (2014) Effects of the economic crisis on health and healthcare in Greece in the literature from 2009 to 2013: a systematic review. Health Policy 115, 111-119. Published online: 8 February 2014.

19. Kondilis E et al. (2013) Economic crisis, restrictive policies, and the population's health and health care: the Greek case. American Journal of Public Health 103, 973-979. Published online June 2013.

20. Ifanti AA et al. (2013) Financial crisis and austerity measures in Greece: their impact on health promotion policies and public health care. Health Policy 113, 8-12.

21. Peragallo MS et al. (2011) Evaluation of cancer surveillance completeness among the Italian army personnel, by capture-recapture methodology. Cancer Epidemiology 35, 132-138. Published online: 17 July 2010.

22. Ungcharoen $\mathbf{R}$ and Ingsrisawang $\mathbf{L}$ (2015) Capture-recapture to estimate completeness of pulmonary tuberculosis with two sources. 17th International Conference on Mathematics, Statistics and Computational Sciences, Tokyo, Japan, 28-29 May 2015. World Academy of Science, Engineering and Technology. International Journal of Mathematical and Computational Sciences 9(5). Available at http://waset.org/pdf/books/? $\mathrm{id}=23818$ \&pageNumber $=6$ (accessed 10 February 2018).

23. Brittain S and Böhning D. (2009) Estimators in capture-recapture studies with two sources. AStA Advances in Statistical Analysis 93, 23-47. Published online: 21 October 2008.

24. Chao A (1987) Estimating the population size for capture-recapture data with unequal catchability. Biometrics 43, 783-791.

25. Armitage P and Berry G. (1994) Statistical Methods in Medical Research, 3rd Edn. Oxford, Boston: Blackwell Scientific Publications.

26. Gill GV, Ismail AA and Beeching NJ (2001) The use of capture-recapture techniques in determining the prevalence of type 2 diabetes. QJ Med 94, 341-346. Published online: 1 July 2001.
27. Brenner H, Stegmaier C and Ziegler H (1995) Estimating completeness of cancer registration: an empirical evaluation of the two source capture-recapture approach in Germany. Journal of Epidemiology and Community Health 49, 426-430.

28. German RR et al. (2001) Updated guidelines for evaluating public health surveillance systems: recommendations from the Guidelines Working Group. MMWR. Recommendations and Reports 50, 1-35.

29. Hall HI et al. (2006) Assessing the completeness of reporting of human immunodeficiency virus diagnoses in 2002-2003: capture-recapture methods. American Journal of Epidemiology 164, 391-397. Published online: 13 June 2006.

30. Hook EB and Regal RR (1995) Capture-recapture methods in epidemiology: methods and limitations. Epidemiologic Reviews 17, 243-264.

31. Brenner H (1995) Use and limitations of the capture-recapture method in disease monitoring with two dependent sources. Epidemiology 6, 42-48.

32. Jelastopulu E, Merekoulias G and Alexopoulos EC (2010) Underreporting of communicable diseases in the prefecture of Achaia, western Greece, 1999-2004 - missed opportunities for early intervention. Euro Surveillance 15, 19579. Published online: 27 May 2010.

33. Gkogka E et al. (2011) Risk-based estimate of effect of foodborne diseases on public health, Greece. Emerging Infectious Diseases 17, 1581-1590. Published online: 9 September 2011.

34. Pérez-Ciordia I et al. (2002) Salmonella enteritis in Huesca: 1996-1999. (In Spanish). Enfermedades Infecciosas y Microbiología Clínica 20, 16-21.

35. Jansson A, Arneborn M and Ekdahl K (2005) Sensitivity of the Swedish statutory surveillance system for communicable diseases 1998-2002, assessed by the capture-recapture method. Epidemiology and Infection 133, 401-407. Published online: 2 February 2005.

36. Haagsma JA et al. (2013) Community incidence of pathogen-specific gastroenteritis: reconstructing the surveillance pyramid for seven pathogens in seven European Union member states. Epidemiology and Infection 141, 1625-1639. Published online: 27 September 2012.

37. Brabazon ED et al. (2008 Under-reporting of notifiable infectious disease hospitalizations in a health board region in Ireland: room for improvement? Epidemiology and Infection 136, 241-247. Published online: 30 March 2007.

38. Amaku M et al. (2017) Estimating the prevalence of infectious diseases from under-reported age-dependent compulsorily notification databases. Theoretical Biology and Medical Modelling 14, 23. Published online: 12 December 2017.

39. Doyle TJ, Glynn MK and Groseclose SL (2002) Completeness of notifiable infectious disease reporting in the United States: an analytical literature review. American Journal of Epidemiology 155, 866-874.

40. Janati A et al. (2015) Communicable disease reporting systems in the world: a systematic review article. Iranian Journal of Public Health 44, 1453-1465.

41. Van Hest NAH et al. (2008) Incidence and completeness of notification of Legionnaires' disease in the Netherlands: covariate capture-recapture analysis acknowledging regional differences. Epidemiology and Infection 136, 540-550. Published online: 22 June 2007.

42. Masters R et al. (2017) Return on investment of public health interventions: a systematic review. Journal of Epidemiology and Community Health 71, 827-834. Published online: 7 March 2017. 